\title{
Plasma protoporphyrin IX following administration of 5-aminolevulinic acid as a potential tumor marker
}

\author{
URARA OTA $^{1}$, HIDEO FUKUHARA ${ }^{2}$, MASAHIRO ISHIZUKA ${ }^{1}$, FUMINORI ABE $^{1}$, CHIAKI KAWADA $^{2}$, \\ KENJI TAMURA ${ }^{2}$, TOHRU TANAKA ${ }^{1}$, KEIJI INOUE ${ }^{2}$, SHUN-ICHIRO OGURA $^{3}$ and TARO SHUIN ${ }^{2}$ \\ ${ }^{1}$ SBI Pharmaceuticals Co., Ltd., Izumi Garden Tower 20F, 1-6-1, Tokyo 106-6020; ${ }^{2}$ Department of Urology, \\ Kochi Medical School, Nankoku, Kochi 783-8505; ${ }^{3}$ Department of Bioengineering, Graduate School of Bioscience \\ and Biotechnology, Tokyo Institute of Technology, Yokohama, Kanagawa 226-8501, Japan
}

Received September 29, 2014; Accepted March 3, 2015

DOI: $10.3892 / \mathrm{mco} .2015 .549$

\begin{abstract}
Exogenously administered 5-aminolevulinic acid (ALA) is metabolized to protoporphyrin IX (PpIX), which specifically accumulates in cancer cells and emits red fluorescence by blue light irradiation. These phenomena are applied for the intraoperative diagnosis of cancer. Based on the fact that accumulated PpIX in cancer cells is exported extracellularly via the ATP-binding cassette transporter G2, we hypothesized that the measurement of plasma PpIX concentrations could be applied as a tumor marker for cancer screening. In the present study, the use of plasma samples from bladder cancer patients were evaluated as a tumor marker. ALA, $1.0 \mathrm{~g}$, was orally administered to bladder cancer patients and healthy adults. The plasma concentration of PpIX was measured using a high-performance liquid chromatography system. The plasma PpIX concentration following ALA administration was significantly higher in bladder cancer patients than that in the healthy adults, suggesting the effectiveness of plasma PpIX analysis following ALA administration for cancer screening. Additionally, $4 \mathrm{~h}$ after ALA administration, plasma PpIX showed high sensitivity (94.4\%) and high specificity (80.0\%).
\end{abstract}

\section{Introduction}

Exogenously administered 5-aminolevulinic acid (ALA) is metabolized through the assembly of eight ALA molecules to a heme precursor protoporphyrin IX (PpIX), and subsequently

Correspondence to: Professor Shun-Ichiro Ogura, Department of Bioengineering, Graduate School of Bioscience and Biotechnology, Tokyo Institute of Technology, 4259 B47 Nagatsuta, Yokohama, Kanagawa 226-8501, Japan

E-mail: sogura@bio.titech.ac.jp

Abbreviations: ALA, 5-aminolevulinic acid; ABCG2, adenosine triphosphate-binding cassette transporter G2; PpIX, protoporphyrin IX

Key words: 5-aminolevulinic acid, protoporphyrin IX, tumor marker, plasma to heme through the insertion of an iron ion. In cancer cells, however, PpIX accumulates due to the Warburg effect, abnormalities in iron metabolism and errors in iron insertion (1). As PpIX is a fluorescent compound, PpIX in cancer is visualized as a useful intraoperative diagnostic agent. This procedure is known as photodynamic diagnosis (PDD) using ALA (2). In Europe and Japan, ALA has been approved as an intraoperative diagnostic agent for brain tumors. Photodynamic diagnosis can be applied not only to brain tumors, but also to various types of cancer, including those of the bladder (3), prostate $(4,5)$, stomach $(6)$ and colon $(7)$, and thus, clinical studies are currently underway to expand its application.

Specific PpIX accumulation in cancer cells following ALA administration remains intracellular for $\sim 10 \mathrm{~h}$ before being exported extracellularly by adenosine triphosphate-binding cassette transporter G2 (ABCG2) $(1,8)$. Our previous study indicated that the measurement of changes in urinary porphyrin metabolites, such as uroporphyrins and coproporphyrins, following ALA administration could be useful in screening for bladder cancer (9). Such changes are possibly attributed to the fact that cancer cells have abnormalities in heme metabolism. However, P PIX is known to be a major porphyrin metabolite accumulating in tumor tissue following ALA administration. Based on these findings, we hypothesized that the measurement of plasma concentration of PpIX, a molecule that is released from the tumor tissue into the plasma may provide more direct information than that of urinary porphyrin metabolites. Therefore, changes in plasma concentrations of porphyrin metabolites were measured, particularly PpIX, following the administration of ALA and evaluated the effectiveness of this assay as a screening test for cancer patients.

\section{Materials and methods}

Materials. ALA hydrochloride was used (1.0 g; Cosmo Bio Co., Tokyo, Japan). Oral ALA solution was prepared by dissolving ALA in a 5\% glucose solution and was ingested $3 \mathrm{~h}$ before endoscopic examination.

Subjects and experimental protocol. Similar to our previous study (9), the ingestion of ALA and PDD procedures were approved by the Ethics Committee of Kochi Medical 
School (Kochi, Japan) on December 26, 2006 (nos. 18-27). The present study involved 73 patients with bladder cancer, consisting of the patients described in the previous study and 7 additional patients, and 25 healthy adults consisting of the healthy subjects included in the previous study and 5 additional subjects in whom no primary or recurrent cancer had been detected by transurethral biopsy of the bladder for 2-3.5 years. The patients received a prior explanation of the study and provided written informed consent. The background characteristics of the patients are shown in Table I. A brief summary of the protocol is as follows: ALA (1.0 g) was administered orally $3 \mathrm{~h}$ before endoscopic examination. Peripheral blood $(5 \mathrm{ml})$ was collected in EDTA-2Na tubes at 4 and $8 \mathrm{~h}$ after ALA administration and centrifuged to obtain plasma for high-performance liquid chromatography (HPLC) analysis of ALA and porphyrin metabolites, including uroporphyrin I (UPI), UPIII, coproporphyrin I (CPI), CPIII and PpIX.

\section{HPLC analysis}

Determination of ALA. Plasma ALA was determined using the method of Endo et al (10), with appropriate modifications. Briefly, $0.2 \mathrm{ml}$ of plasma was mixed with $25 \% \mathrm{w} / \mathrm{v}$ TCA solution by agitation and was centrifuged. Subsequently, $0.01 \mathrm{ml}$ of the resulting supernatant was transferred to a test tube, incubated with $0.24 \mathrm{ml}$ of Milli-Q water, $0.25 \mathrm{ml}$ of $200 \mathrm{mM}$ acetic acid-sodium acetate buffer ( $\mathrm{pH} 3.8), 1.25 \mathrm{ml}$ of solution A ( $68.4 \mathrm{mM}$ sodium chloride containing $15 \% \mathrm{v} / \mathrm{v}$ acetylacetone and $10 \% \mathrm{v} / \mathrm{v}$ ethanol) and $0.25 \mathrm{ml}$ of solution B (3.3\% w/w formaldehyde) in boiling water for $15 \mathrm{~min}$, and immediately cooled in ice water. This reaction mixture was applied to a HPLC system (Inertsil ODS-3, $5 \mu \mathrm{m}, 4.6 \times 150 \mathrm{~mm}$; GL Sciences, Tokyo, Japan) for analysis.

Determination of porphyrin metabolites. Plasma UPI, UPIII, CPIand CPIII were determined using the method of Kondo et al (11) with partial modification. Plasma $(0.1 \mathrm{ml})$ was agitated for $1 \mathrm{~min}$ with $0.8 \mathrm{ml}$ of a mixture of ethyl acetate-acetic acid-25\% w/v TCA (8:1:1 by vol.) and centrifuged at 20,000 x g for $3 \mathrm{~min}$ at $4^{\circ} \mathrm{C}$. After collecting the supernatant in a glass tube, the resulting precipitate was agitated for $1 \mathrm{~min}$ with $0.2 \mathrm{ml}$ of ethyl acetate-acetic acid solution (4:1 by vol.) and centrifuged to obtain the supernatant (this step was repeated twice). The three batches of supernatant were combined, incubated at $40^{\circ} \mathrm{C}$ in a heating block, and evaporated to dryness under nitrogen gas. The porphyrin-containing solid was dissolved in $0.2 \mathrm{ml}$ of acetonitrile- $50 \% \mathrm{v} / \mathrm{v}$ acetic acid solution (4:1 by vol.) and analyzed with a HPLC system (Symmetry C18, $5 \mu \mathrm{m}$, 3.9x150 mm; Ex. 404 nm, Em. 620 nm; Waters, Milford, MA, USA). The elution conditions were as follows: Solvent A, $1 \mathrm{M}$ ammonium acetate containing $10.5 \%$ acetonitrile ( $\mathrm{pH} 5.2$ ); solvent B, $50 \mathrm{mM}$ ammonium acetate containing $80 \%$ acetonitrile (pH 5.2); and gradient, $100 \%$ solvent $\mathrm{A}$ at $1 \mathrm{ml} / \mathrm{min}$ for 10 min, followed by a linear increase of solvent $\mathrm{B}$ from 20 to $27.2 \%$ over $14.5 \mathrm{~min}$ and $100 \%$ solvent B for $10 \mathrm{~min}$.

For the determination of PpIX, $0.1 \mathrm{ml}$ of plasma was vigorously agitated for $30 \mathrm{sec}$ with $0.01 \mathrm{ml}$ of $50 \% \mathrm{v} / \mathrm{v}$ acetic acid and $0.3 \mathrm{ml}$ of $N, N$-dimethylformamide-2-propanol (DMF-IPA) solution (100:1 by vol.) and centrifuged at 20,000 x g for $5 \mathrm{~min}$ at $4^{\circ} \mathrm{C}$ to collect supernatant. The precipitate was extracted with $0.15 \mathrm{ml}$ of DMF-IPA solution by repeating the same procedures as above. The two batches of supernatant were mixed and analyzed with an HPLC system using a Capcell Pak C18 UG120 column (5 $\mu \mathrm{m}, 4.6 \times 150 \mathrm{~mm}$, Shiseido, Tokyo, Japan), mobile phase of $10 \mathrm{mM}$ tetrabutylammonium hydroxide solution ( $\mathrm{pH} 7.5$ ) (flow rate, $1.0 \mathrm{ml} / \mathrm{min}$; elution temperature, $40^{\circ} \mathrm{C}$ ), and fluorescence detector (Ex. $400 \mathrm{~nm}$, Em. $630 \mathrm{~nm}$ ).

Statistical analysis. Mann-Whitney's U and Steel-Dwass' tests were used for the analysis. $\mathrm{P}<0.05$ was considered to indicate a significant concentration. Diagnostic efficacy was evaluated using the area under the curve (AUC) on receiver-operating characteristics (ROC) analysis.

\section{Results}

Plasma concentrations of ALA, PpIX and porphyrins. The background characteristics of patients with bladder cancer are summarized in Table I. Measurements of the plasma ALA, PpIX and total porphyrin concentrations at 4 and $8 \mathrm{~h}$ after the oral administration of ALA in these cancer patients and healthy adults are shown in Fig. 1. The median plasma ALA concentration at 4 and $8 \mathrm{~h}$ after ALA administration was $17.0(4.3-66.4)$ and $3.8(0-8.2) \mu \mathrm{mol} / 1$, respectively, in healthy adults (HA group), compared to 53.0 (2.6-124.9) and $6.4(0.4-45.9) \mu \mathrm{mol} / 1$, respectively in bladder cancer patients (BC group), demonstrating that plasma ALA concentrations were significantly higher in the BC group compared to the HA group at 4 and $8 \mathrm{~h}$ after ALA administration (Fig. 1A). The median plasma PpIX concentrations at 4 and $8 \mathrm{~h}$ after ALA administration was $2.8(0.8-180.9)$ and $0.2(0-187.6) \mathrm{nmol} / \mathrm{l}$, respectively, in the HA group, compared to 69.1 (2.8-224.5) and 41.8 (4.0-229.9) nmol/l, respectively, in the BC group. Similar to the ALA concentrations, plasma PpIX concentrations were significantly higher in the BC compared with in the HA group at 4 and $8 \mathrm{~h}$ after ALA administration (Fig. 1B). Additionally, similar to the PpIX concentrations, total porphyrin concentrations in plasma were also significantly higher in the BC compared with the HA group (Fig. 1C).

\section{Stages of bladder cancer progression and plasma concentra-} tions of ALA and PpIX. For the BC group, the data shown in Fig. 1 were classified into four groups according to the stage of bladder cancer (pTa, pTis, pT1 and pT2-4), and compared with those for the HA group (Fig. 2). The median plasma ALA concentration at $4 \mathrm{~h}$ after ALA administration was 50.6 (2.6-109.2), 69.3 (35.7-122.7), 62.3 (27.1-105.8), and $49.7(12.2-124.9) \mu \mathrm{mol} / 1$ in the pTa,pTis, pT1, and pT2-4 groups, respectively. The median plasma PpIX concentrations at $4 \mathrm{~h}$ after ALA administration was 65.2 (2.8-224.5), 82.1 (55.4-216.3), $65.4(26.5-160.6)$, and $68.9(25.3-158.3) \mathrm{nmol} / \mathrm{l}$ in the pTa, pTis, pT1, and pT2-4 groups, respectively. Plasma concentrations of ALA and PpIX at $4 \mathrm{~h}$ after ALA administration were significantly increased in all the stages of bladder cancer compared with those in the HA group. However, there were no marked differences in plasma concentrations of ALA and PpIX concentration across the tumor stages. A similar tendency of ALA and PpIX plasma concentrations were observed $8 \mathrm{~h}$ after ALA administration. 
Table I. Characteristics of the bladder cancer patients.

TNM staging

\begin{tabular}{lcccc}
\cline { 2 - 4 } Characteristics & pTa & pTis & pT1 & pT2-4 \\
\hline Age, median years (range) & $70.5(49.1-85.0)$ & $72.5(48.0-86.0)$ & $76.0(64.0-90.0)$ & $79.0(53.0-87.0)$ \\
Male/female, $\mathrm{n}$ & $34 / 5$ & $10 / 2$ & $8 / 3$ & $9 / 2$
\end{tabular}

Data from 73 bladder cancer patients were divided into 4 groups according to the stage of disease progression: pTa, pTis, pT1 and pT2-4. TNM, tumor-node-metastasis.

A

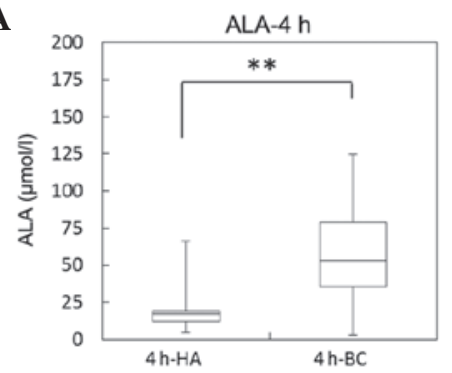

D

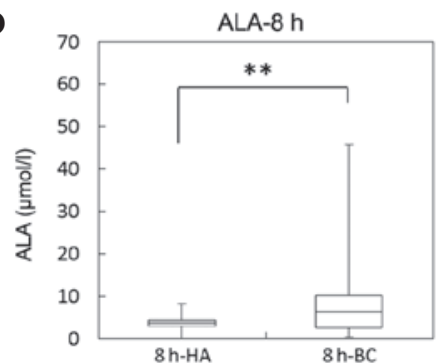

B

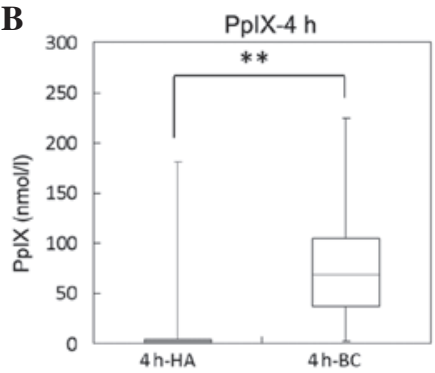

E

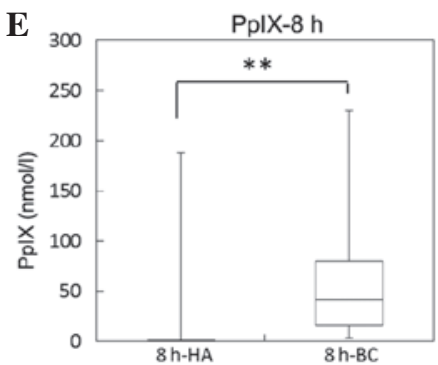

C

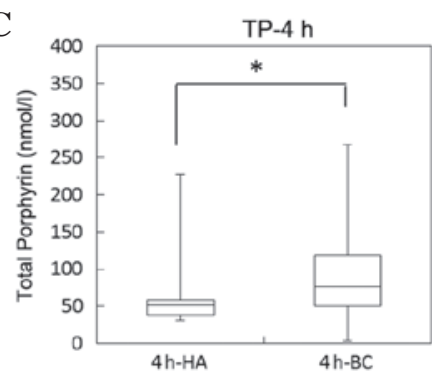

F

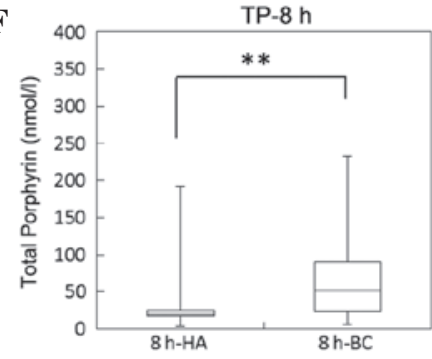

Figure 1. Plasma 5-aminolevulinic acid (ALA), protoporphyrin IX (PpIX) and total porphyrin concentrations at 4 and $8 \mathrm{~h}$ after ALA administration in healthy adults and bladder cancer patients. An oral dose of $1.0 \mathrm{~g}$ of ALA hydrochloride was administered to healthy adults (HA, $\mathrm{n}=25)$ and patients with bladder cancer (BC, $\mathrm{n}=73$ ). The plasma concentrations of ALA, PpIX and total porphyrin at 4 and $8 \mathrm{~h}$ after ALA administration were measured by high-performance liquid chromatography. The (A-C) upper panel shows measurements at $4 \mathrm{~h}$ and the (D-F) lower panel at $8 \mathrm{~h}$ after ALA administration. ${ }^{*} \mathrm{P}<0.05$ and ${ }^{* * *} \mathrm{P}<0.01$ by Mann-Whitney's U-test.

A

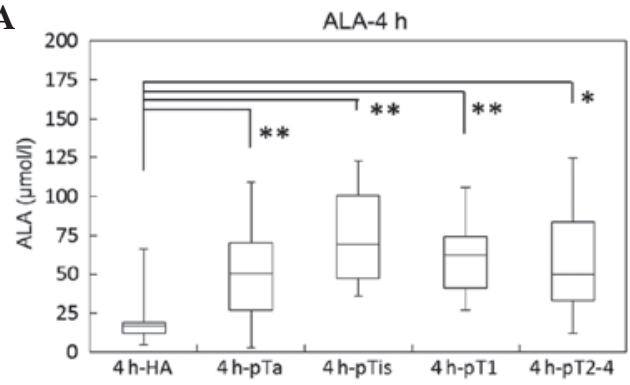

C

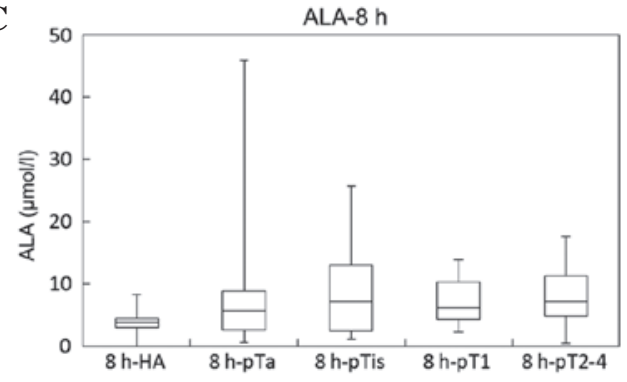

$\mathbf{B}$

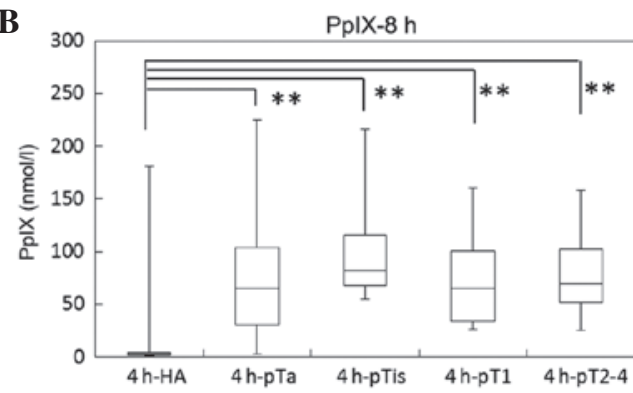

D

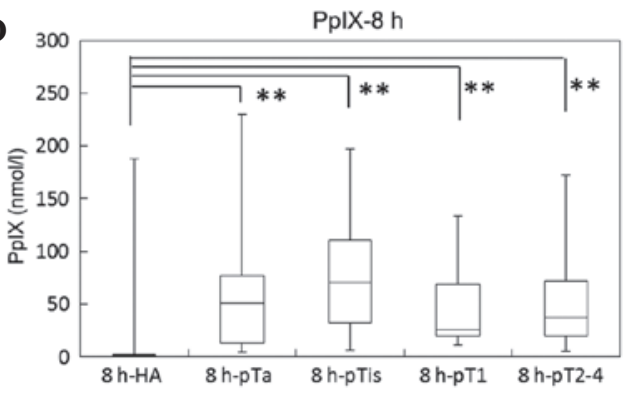

Figure 2. Plasma concentrations of 5-aminolevulinic acid (ALA) and protoporphyrin IX (PpIX) by stage of bladder cancer. An oral dose of $1.0 \mathrm{~g}$ of ALA hydrochloride was administered to healthy adults $(\mathrm{HA}, \mathrm{n}=25)$ and bladder cancer patients $(\mathrm{n}=73)$ who were classified into four groups according to the stage of disease progression: pTa, pTis, pT1 and pT2-4. The plasma concentrations of ALA and PpIX at (A and B) 4 and (C and D) 8 h after ALA administration were measured by high-performance liquid chromatography. " $\mathrm{P}<0.05$ and ${ }^{* *} \mathrm{P}<0.01$ by Steel-Dwass's test. 
A

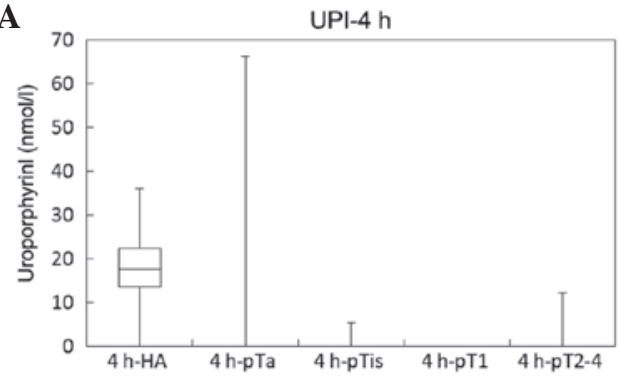

C

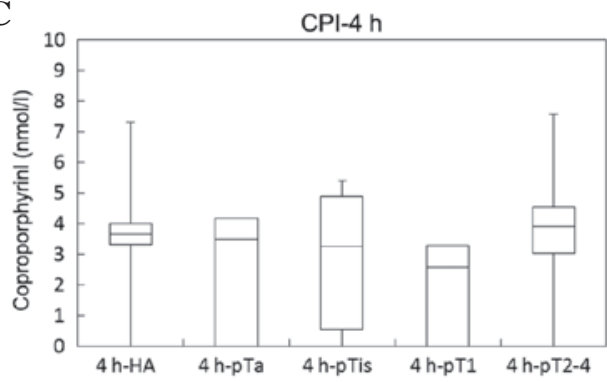

B

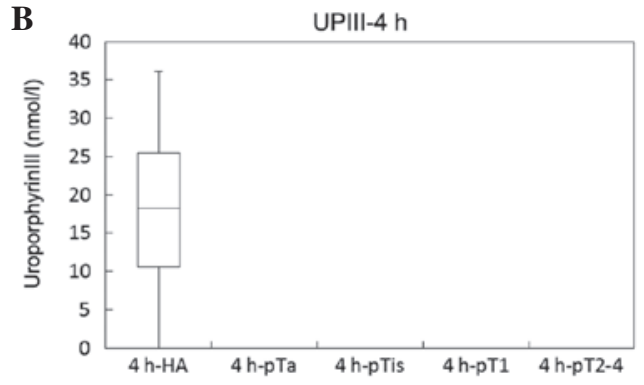

D

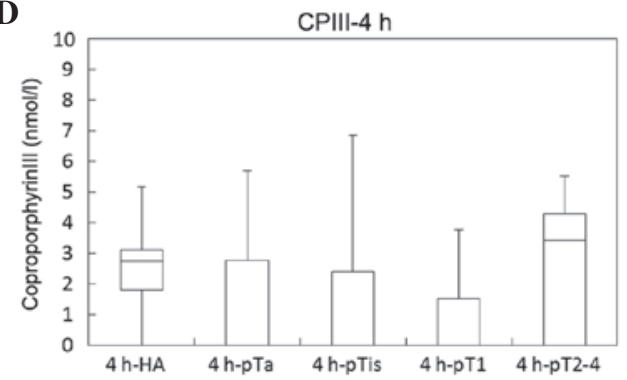

Figure 3. Plasma concentrations of porphyrin metabolites (UPI, UPIII, CPI and CPIII) by stage of bladder cancer An oral dose of $1.0 \mathrm{~g}$ of ALA hydrochloride was administered to healthy adults ( $\mathrm{HA}, \mathrm{n}=25)$ and bladder cancer patients $(\mathrm{n}=73)$ who were classified into 4 groups according to the stage of disease progression: pTa, pTis, pT1 and pT2-4. The plasma concentrations of porphyrin metabolites (UPI, UPIII, CPI and CPIII) at (A and B) 4 and (C and D) 8 h after ALA administration were measured by HPLC. "P<0.05 and ${ }^{* *} \mathrm{P}<0.01$ by Steel-Dwass's test. UP, uroporphyrin; CP, coproporphyrin; ALA, 5-aminolevulinic acid; HPLC, high-performance liquid chromatography.

A

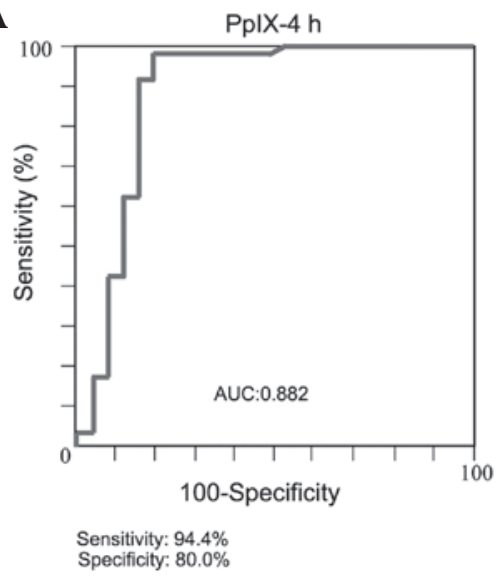

B

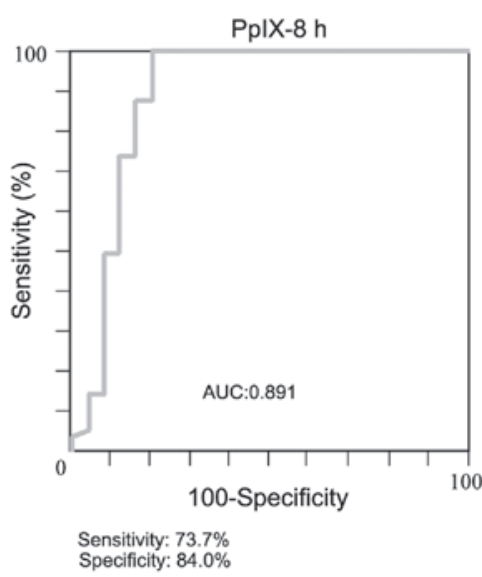

Figure 4. Receiver-operating characteristics (ROC) curves for plasma protoporphyrin IX (PpIX) concentrations at (A) 4 and (B) 8 h after 5-aminolevulinic acid (ALA) administration. An oral dose of $1.0 \mathrm{~g}$ of ALA hydrochloride was administered to healthy adults (HA, $\mathrm{n}=25)$ and patients with bladder cancer (BC, $\mathrm{n}=73$ ). An ROC analysis was performed of plasma concentrations of PpIX measured at 4 and $8 \mathrm{~h}$ after ALA administration using a cut-off value of $20 \mathrm{nmol} / 1$. AUC, area under the curve.

Plasma concentrations of UPI, UPIII, CPI and CPIII. Plasma concentrations of porphyrin metabolites, such as UPI, UPIII, CPI and CPIII, following ALA administration were analyzed (Fig. 3). The median concentrations of these porphyrin metabolites in plasma were markedly lower than those in urine, corresponding to only $1 / 60-1 / 1,800$ of the urinary concentrations described in our previous study (9).

Analysis of ROC curves. An ROC analysis was performed of plasma PpIX concentrations (Fig. 4). A cut-off value of $20 \mathrm{nmol} / \mathrm{l}$ was determined by the ROC analysis. The AUC, sensitivity and specificity of the PpIX measurement at $4 \mathrm{~h}$ post-administration were $0.882,94.4$ and $80.0 \%$, respectively, whereas those of the measurement at $8 \mathrm{~h}$ post-administration were $0.891,73.7$ and $84.0 \%$, respectively.

\section{Discussion}

Patients with bladder cancer, regardless of the stage of progression, showed significantly higher plasma PpIX concentrations following ALA administration compared to healthy adults, demonstrating the potential usefulness of the plasma PpIX concentration following ALA administration in screening for bladder cancer. In addition, $4 \mathrm{~h}$ after plasma PpIX concentration showed a greater diagnostic efficacy than $8 \mathrm{~h}$ after plasma PpIX concentration. To the best of our knowledge, there is no tumor marker for bladder cancer that can be detected in plasma. Therefore, this is the first study of a plasma tumor marker for bladder cancer.

There are marked differences in the urinary excretion of UPs and CPs following ALA administration between 
healthy subjects and bladder cancer patients. The plasma concentrations of UPs and CPs measured in the present study were markedly lower, corresponding to only $1 / 60-1 / 1,800$ of the reported urinary concentrations, and were $<1 / 10$ of the plasma PpIX concentrations. There was a $>10$ times difference in the plasma PpIX concentrations between healthy adults and bladder cancer patients. Although it is difficult to compare the suitability of urine and plasma samples for cancer screening, the measurement of plasma PpIX could be more advantageous than that of urinary porphyrins in terms of sensitivity.

In the present study, cancer patients showed significantly higher plasma ALA concentrations compared to the healthy adults. This is possibly due to potential differences in the metabolic rate of ALA between normal and cancer cells that have abnormalities in their overall heme metabolism. However, considering that the examined cancer patients were older than the healthy subjects and thus, may have a decreased metabolism and a reduced metabolic rate of ALA, the present findings warrant further, careful investigation.

In cancer cells, PpIX is considered to be generated in the mitochondria. Increased plasma PpIX in cancer patients possibly originates from cancer cells, in which PpIX is generated and exported through the transporter. Therefore, plasma PpIX may provide direct evidence for the presence of tumors.

The measurement of plasma concentrations of PpIX allows a more direct index of the presence of tumors, as well as advantages in sensitivity and in that no data correction is necessary, whereas measurement of the urinary excretion of UPs and CPs is less invasive than a blood test. For these reasons, it would be favorable to apply the urine test to a mass screening program, such as health examinations, and use the blood test for individuals with abnormal urine on screening and those who are at a higher risk of cancer to test, such as for the recurrence of cancer.

Although the present study focused on bladder cancer, the principle that PpIX accumulates specifically in cancer cells following ALA administration is common to almost all types of cancer. In order to optimize the assay conditions, further studies are required to confirm these findings in various types of cancer and determine the optimal dose of ALA.

\section{Acknowledgements}

The present study was supported by the Adaptable and Seamless Technology Transfer Program through target-driven research and development from the Japan Science and Technology Agency and the Grant-in-Aid for Scientific Research (C) (grant nos. 26430141 and 21592050) from the Ministry of Education, Culture, Sports, Science and Technology.

\section{References}

1. Ishizuka M, Abe F, Sano Y, et al: Novel development of 5-aminolevurinic acid (ALA) in cancer diagnoses and therapy. Int Immunopharmacol 11: 358-365, 2011.

2. Stummer W, Pichlmeier U, Meinel T, et al: Fluorescence-guided surgery with 5-aminolevulinic acid for resection of malignant glioma: a randomised controlledmulticentre phase III trial. Lancet Oncol 7: 392-401, 2006.

3. Inoue K, Fukuhara H, Shimamoto T, et al: Comparison between intravesical and oral administration of 5-aminolevulinic acid in the clinical benefit of photodynamic diagnosis for nonmuscle invasive bladder cancer. Cancer 118: 1062-1074, 2012.

4. Inoue K, Ashida S, Fukuhara H, et al: Application of 5-aminolevulinic acid-mediated photodynamic diagnosis to robot-assisted laparoscopic radical prostatectomy. Urology 82: 1175-1178, 2013.

5. Fukuhara $\mathrm{H}$, Inoue $\mathrm{K}$, Satake $\mathrm{H}$, et al: Photodynamic diagnosis of positive margin during radical prostatectomy: preliminary experience with 5-aminolevulinic acid. Int J Urol 18: 585-591, 2011.

6. Namikawa T, Inoue K, Uemura S, et al: Photodynamic diagnosis using 5-aminolevulinic acid during gastrectomy for gastric cancer. J Surg Oncol 109: 213-217, 2014.

7. Kondo Y, Murayama Y, Konishi $\mathrm{H}$, et al: Fluorescent detection of peritoneal metastasis in human colorectal cancer using 5-aminolevulinic acid. Int J Oncol 45: 41-46, 2014

8. Hagiya Y, Fukuhara H, Matsumoto K, et al: Expression levels of PEPT1 and ABCG2 play key roles in 5-aminolevulinic acid (ALA)-induced tumor-specific protoporphyrin IX (PpIX) accumulation in bladder cancer. Photodiagnosis Photodyn Ther 10: 288-295, 2013

9. Inoue $\mathrm{K}$, Ota U, Ishizuka M, et al: Porphyrins as urinary biomarkers for bladder cancer after 5-aminolevulinic acid (ALA) administration: the potential of photodynamic screening for tumors. Photodiagnosis Photodyn Ther 10: 484-489, 2013.

10. Endo Y, Okayama A, Endo G, et al: Improvement of urinary $\delta$-aminolevulinic acid determination by HPLC and fluorescence detection using condensing reaction with acetylacetone and formaldehyde. Jpn J Ind Health 36: 49-56, 1994.

11. Kondo M, Sekine K and Takara S: Occurrence of anemia and alterations in porphyrin metabolism in chronic hemodialysis patients. Porphyrins 3: 297-302, 1994. 